\title{
The rugged landscape of drug design
}

\section{How can we get more therapies into preclinical testing and increase the proportion that succeed in preclinical testing? How can we increase the efficacy of therapies? How can we ensure that therapies are developed for rare diseases?}

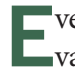
very bridge has its troll. That is to say, wherever there is something of value, you will find someone passionate about it with whom you will need to deal individually. By finding the right person, it is possible to set up deals of mutual and transferrable benefit. For the patient, it is his life that is valuable; for the researcher, her ideas and data; for the biotechnologist, his start-up; for the investor, her capital. Why, then, should we expect people working in pharmaceutical companies to think or act any differently? Their value lies in proprietary medicinal chemistry and extensive infrastructure for quality control and navigation of clinical trials. Where, then, are the passionate pharma decision makers willing to make the deals that will fill the preclinical pipeline with promising new therapies?

One possibility, that big pharma is big enough to carry out the whole process of drug development internally, does not stand scrutiny. It seems that the process of autophagy-the self-consuming mergers that pharmaceutical companies have undergone to preserve their value to shareholders-has impaired the flow of new drugs reaching preclinical approval, despite increased spending on research and development. If the landscape of drug design were smooth, the experience of successfully developing one drug would inform the next one. However, at the early stages of development, successes are represented by a series of isolated peaks with almost no connection from one to the next. If, because of this unpredictable risk, investment by pharma and venture capital is drying up for early development activities, someone else needs to step up to ensure that the drug pipeline is filled.

These were the urgent issues discussed by an expert panel convened at the Amyotrophic Lateral Sclerosis Therapy Development Institute (ALS TDI: http://www.als.net/summit/Schedule.aspx). Leaders from the fields of biotechnology, venture capital and government had the opportunity to express their visions for the future and confront the reality that we are taking a hard road in hard financial times in which it will be difficult to achieve drug discovery and translation. This view was simply unacceptable to the many people with progressive motor neuron disease in the audience facing their current odds. There are at present 156 potential drugs under investigation for ALS, and barely one therapy in 1,000 passes the preclinical testing hurdle. There is only one therapy approved by the US Food and Drug Administration for ALS. The drug, riluzole, provides only a very limited delay in the progression of the disease. The heterogeneity of this relatively rare disease, with 19 genes and several possible environmental factors implicated in the risk of onset and an extremely variable rate of progression, is a major obstacle to drug development. In contrast, Biogen Idec alone has eight drugs in phase 2 or 3 trials for multiple sclerosis, so it is likely that there will soon be some benefit to people with this condition.

Rarity of a disease need not be a barrier to drug development. When the time is right, all the components needed for drug development fall into place. Some 27 years ago, teams led by Genzyme pioneered the development of recombinant enzyme replacement therapies for lysosomal storage diseases, some of the rarest and most expensive of rare disease to treat. It was then so important to demonstrate that recombinant biologics could be manufactured and used clinically that rarity and the economic reality of delivery were no obstacles. The ALS area apparently has just enough money available so that the unmet need will be addressed. "Why tweak this?" noted a panelist rhetorically; at ALS TDI new approaches are being tried. "They are not normal players, they have found unique funding and opportunities, and the decisions taken are rather different."

Long-term government funding can target drugs to diseases with low prevalence and high risk of translational failure. In this role, we have the intramural NIH Center for Translational Therapeutics program on Therapeutics for Rare and Neglected Diseases (http://nctt.nih.gov/trnd) and the proposed National Center for Advancing Translational Sciences (http://www.nih.gov/about/director/ncats/NCATSbudget.pdf). The consensus of the panel was that although government funding is key to basic research, often serves a catalytic function and might be targeted to help prevent loss of momentum in the translational process, commercial incentives will ultimately still work better if each step of drug development can be made an attractive target for funding.

Decision making in the private sector is limited by the need for quick return on capital, but standards of quality, teamwork and deliverables are very much a part of corporate culture. These virtues are pragmatic rather than idealistic, however. Concepts of precompetitive data sharing and simple contracts may work when there is nothing at stake, but anything of value needs champions. The coordinates and nomenclature of the human genome work like a universal language to enable life science research, as do the uniform structural descriptors for chemical molecules. These conventions can be adopted, but it will take more than exhortation to change current attitudes to datasets and ideas with commercial value. It will take truly exceptional incentives possibly exceeding the potential dollar value of the resource. Even then, such initiatives may not work because of the need to own and promote the work and its intellectual property.

Early translational development is probably best initiated by academic researchers and biotech companies. If the large pharmaceutical companies that can afford the medicinal chemistry, quality control and full-scale clinical trials find this idea attractive and decide to participate, they will have more drugs to sell. If not, funding will increasingly follow a new model. The missing components are not-for-profit translational facilities modeled on ALS TDI with funds raised by patient advocates, government and venture capital in a mix designed to prevent loss of momentum at each stage of the development process. How, then, should we evaluate ALS TDI as a biotech venture? It is not a company in the traditional sense, and is possibly the only not-for-profit in its space with a focused effort on translation. It should continue doing what it does to extraordinary effect. 